\title{
Hyponatraemia secondary to nivolumab-induced primary adrenal failure
}

\author{
Harris Trainer2, Paul Hulse1, Claire E Higham², Peter Trainer² and Paul Lorigan³ \\ Departments of ${ }^{1}$ Radiology, ${ }^{2}$ Endocrinology, and ${ }^{3}$ Medical Oncology, The Christie NHS Foundation Trust, University \\ of Manchester, Manchester Academic Health Science Centre, Manchester, UK
}

\author{
Correspondence \\ should be addressed \\ to $\mathrm{P}$ Lorigan \\ Email \\ Paul.Lorigan@manchester. \\ ac.uk
}

\section{Summary}

Checkpoint inhibitors, such as ipilimumab and pembrolizumab, have transformed the prognosis for patients with advanced malignant melanoma and squamous non-small-cell lung cancer, and their use will only expand as experience is gained in a variety of other malignancies, for instance, renal and lymphoma. As the use of checkpoint inhibitors increases, so too will the incidence of their unique side effects, termed immune-related adverse events (irAEs), which can affect dermatological, gastrointestinal, hepatic, endocrine and other systems. Nivolumab is a monoclonal antibody that blocks the human programmed death receptor-1 ligand (PD-L1) found on many cancer cells and is licensed for the treatment of advanced malignant melanoma. We describe the first case of nivolumab-induced adrenalitis resulting in primary adrenal failure presenting with hyponatraemia in a 43-year-old man with malignant melanoma. The case highlights the potentially life-threatening complications of checkpoint inhibitors and the need for patient education and awareness of irAEs among the wider clinical community because such side effects require prompt recognition and treatment.

\section{Learning points:}

- Nivolumab can cause primary adrenal insufficiency.

- Not all cases of hyponatraemia in patients with malignancy are due to SIADH.

- Any patient on a checkpoint inhibitor becoming unwell should have serum cortisol urgently measured and if in doubt hydrocortisone therapy should be initiated.

- Although hyponatraemia can occur in patients with ACTH deficiency, the possibility of primary adrenal failure should also be considered and investigated by measurement of renin, aldosterone and ACTH.

- Patients receiving checkpoint inhibitors require education on the potential risks of hypocortisolaemia.

- PET imaging demonstrated bilateral increased activity consistent with an autoimmune adrenalitis.

\section{Background}

Checkpoint inhibitors are immunomodulators that stimulate T-cell-mediated cytotoxicity directed at cancer cell antigens and have transformed the prognosis for patients with advanced melanoma and improved outcomes for patients with advanced renal, non-small-cell lung cancer and Hodgkin's lymphoma $(1,2)$.
Ipilimumab was the first checkpoint inhibitor to be licensed for the treatment of melanoma and functions by binding to and inhibiting the cell-surface-expressed cytotoxic T lymphocyte-associated antigen 4 (CTLA-4), that is responsible for the inhibition of cytotoxic $\mathrm{T}$ lymphocytes (3). Nivolumab and pembrolizumab are 
monoclonal antibodies that block human programmed death receptor-1 ligand (PD-L1) found on many cancer cells, attaching to the human programmed death receptor-1 (PD-1) found on the surface of activated $\mathrm{T}$ cells. The binding of PD-1 to its ligand results in the inactivation of the T-cell. Antibodies that block this interaction allow the immune system to attack the cancer cell. Anti PD-1 antibodies have revolutionised the treatment of melanoma and non-small-cell lung cancer and are being evaluated in a broad range of other cancers.

The use of checkpoint inhibitors in cancer therapy treatment is complicated by numerous mechanism-based toxicities, termed immune-related adverse events (irAEs), affecting dermatological, gastrointestinal, hepatic, endocrine and other systems. However, the biological mechanism by which these irAEs occur is still neither fully understood nor is the predisposition of the various agents to cause different endocrinopathies. Ipilimumab (target CTLA-4) in particular is a well-recognised cause of pituitary dysfunction (hypophysitis and/or hypopituitarism), reported to occur in up to $25 \%$ of patients $(1,4)$, often presenting with multiple anterior pituitary hormone deficiencies. The nature of the pituitary hormone deficiency at presentation is variable with the most common deficiency being in TSH through to complete anterior panhypopituitarism and very rarely diabetes insipidus. Hypopituitarism complicated by adrenal crisis has been reported as the cause of death in a patient on combination therapy with ipilimumab and nivolumab (5). Hypophysitis can result in pituitary enlargement, which may result in mass-effect symptoms such as headache and visual field defects due to optic nerve compression. This is often followed by an 'empty sella' radiologically. Often, however, patients present without neurological symptoms and with general malaise and debility.

In contrast to agents targeting CTLA-4, agents acting on PD-L1 (nivolumab and pembrolizumab) are more commonly associated with thyroid dysfunction, and autoimmune thyroiditis in $9 \%$ of melanoma patients (4), that results in transient hyperthyroidism often followed up by long-term hypothyroidism (4). Hypophysitis with PD-1 inhibitors is rare, occurring in $<1 \%$ of patients. Long-term follow-up of endocrine irAEs suggests that on occasions thyroid function may recover, but that dysfunction of the corticosteroid and gonadal axis is likely to be permanent (6).

\section{Case presentation}

A healthy 43-year-old male presented three years previously with new onset bleeding of a longstanding pigmented lesion of the left shin. A shave excision was undertaken, which histology confirmed as malignant melanoma (Breslow $1.8 \mathrm{~mm}$ ) and subsequently a wide local excision and sentinel node biopsy was performed with two of six nodes containing tumour. There was no evidence of metastatic disease (Stage 3A) on CT staging. The patient had no significant medical history and no family history of melanoma.

A routine FDG PET/CT scan two years after the initial diagnosis showed a new 26 by $21 \mathrm{~mm}$ retroperitoneal para-aortic nodal metastasis and the patient was started on nivolumab $(3 \mathrm{mg} / \mathrm{kg})$ at two weekly intervals. Initial treatment was well tolerated, with follow-up scans showing a reduction in node size.

After four cycles (8 weeks) serum sodium started to fall, and by the end of cycle 5 , it had dropped to $127(135-145 \mathrm{mmol} / \mathrm{L})$. There was an associated four-week history of fatigue, anorexia, weight loss and postural symptoms. Examination was unremarkable, and blood pressure was recorded as $111 / 83 \mathrm{mmHg}$ with no postural drop.

\section{Investigation}

Investigation revealed the following: random urinary sodium $102 \mathrm{mmol} / \mathrm{L}$, random serum cortisol $192 \mathrm{nmol} / \mathrm{L}$, fT4 $17.7 \mathrm{pmol} / \mathrm{L}$, TSH $2.02 \mathrm{U} / \mathrm{L}, \quad \mathrm{LH} 2 \mathrm{U} / \mathrm{L}, \quad F S H 2 \mathrm{U} / \mathrm{L}$, testosterone $6.1 \mathrm{nmol} / \mathrm{L}$, prolactin $148 \mathrm{U} / \mathrm{L}$ and urinary sodium $102 \mathrm{mmol} / \mathrm{L}$. Before the administration of $250 \mu \mathrm{g}$ synacthen cortisol was $186 \mathrm{nmol} / \mathrm{L}$, with postadministration values of 157 and $172 \mathrm{nmol} / \mathrm{L}$, at 30 and 60 min respectively.

\section{Treatment}

The inadequate peak serum cortisol response to synacthen was indicative of cortisol deficiency, and the patient commenced on oral hydrocortisone, initially $20 \mathrm{mg}$ three times daily for three days, which was reduced to a total daily dose of $30 \mathrm{mg}$ per day with improvement in symptoms, but one month later, serum sodium was unchanged at $127 \mathrm{mmol} / \mathrm{L}$.

Subsequently, the patient had an unremarkable MR scan of the pituitary gland, and the results from blood drawn before commencing hydrocortisone revealed ACTH and renin to be elevated at $200 \mathrm{ng} / \mathrm{L}$ 


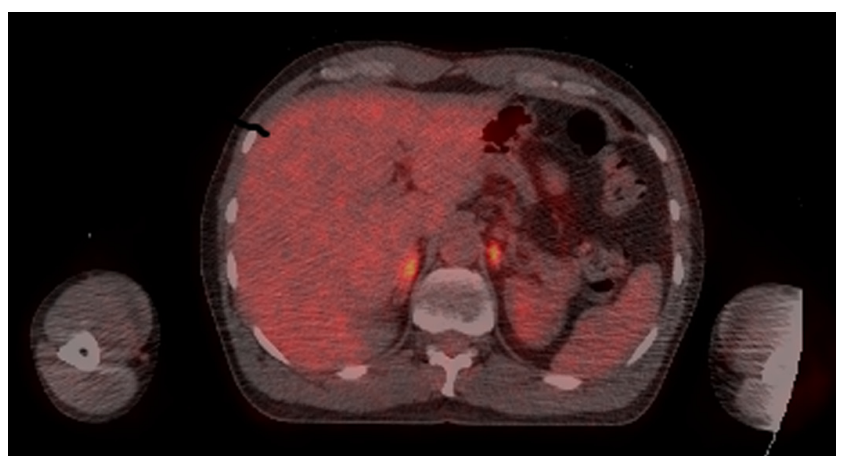

\section{Figure 1}

FDG PET CT scan demonstrating bilateral increased FDG activity in the adrenals (right adrenal SUVmax $=5.2$, left adrenal SUVmax $=5.4$, background liver SUVmax $=2.7$ ).

and $19.8 \mathrm{nmol} / \mathrm{L} / \mathrm{h}$ respectively, with a corresponding aldosterone of $<100 \mathrm{pmol} / \mathrm{L}$ indicative of primary adrenal insufficiency. A routine monitoring PET scan showed a reduction in the size of the para-aortic nodes but uniformly increased FDG activity in both adrenal glands (Fig. 1).

Once established on hydrocortisone and fludrocortisone the patient's symptoms resolved and serum sodium returned to normal. The patient continued nivolumab therapy without interruption.

\section{Outcome and follow-up}

At last review, the patient had completed one year of nivolumab and was entirely well with normal blood biochemistry (serum sodium $141 \mathrm{mmol} / \mathrm{L}, \mathrm{ACTH}$ $10 \mathrm{ng} / \mathrm{mL}$ and renin $1.6 \mathrm{nmol} / \mathrm{L} / \mathrm{h}$ ) on hydrocortisone $30 \mathrm{mg}$ and fludrocortisone $100 \mu \mathrm{g}$, respectively, per day. PET imaging revealed a normal appearance of the adrenal glands and complete resolution of the retroperitoneal lymph node, along with no other evidence of endocrinopathy or other irAEs.

\section{Discussion}

Immunotherapy is rapidly establishing itself as the standard of care for melanoma and second-line management for NSCLS and renal cancer. With the increasing number of agents available and the growing list of indications, their use within clinical practice is set to dramatically increase. These drugs have been shown to cause a unique set of toxicities that most physicians may not have encountered. It is therefore vital that acute care physicians and GPs are educated regarding the recognition and management of such complications.
This is the first report of monotherapy with nivolumab inducing primary adrenal failure. The case illustrates several important learning points. Firstly, there is often an assumption in patients with malignancy that hyponatraemia is secondary to the syndrome of inappropriate anti-diuretic hormone (SIADH). This case highlights the importance of a systematic approach to the investigation of hyponatraemia. Hypocortisolaemia must always be excluded as a potential life-threatening cause of hyponatraemia. The initial mid-morning cortisol of $192 \mathrm{nmol} / \mathrm{L}$ could be regarded as unremarkable, but the confirmation of hypoadrenalism by the absence of any cortisol increment in response to synacthen emphasises that a low threshold is required for performing short synacthen tests.

In the acutely unwell patient, blood should be drawn for the measurement of a random serum cortisol, preferably with a paired plasma ACTH, and glucocorticoid replacement therapy commenced immediately. Confirmation, if necessary, of cortisol deficiency and detailed investigation of the aetiology can be undertaken electively once the patient is well.

In view of the normal prolactin, thyroid function, gonadotrophins and testosterone, the initial assumption was that the patient had developed isolated ACTH deficiency secondary to checkpoint inhibitor-induced hypophysitis, a well-recognised complication of ipilimumab (4). However, the failure of the hyponatraemia to fully resolve with hydrocortisone monotherapy plus the subsequent availability of adrenal and pituitary imaging, combined with elevated ACTH and renin levels were consistent with aldosterone deficiency and primary adrenal failure. Serum sodium and plasma renin promptly returned to normal with the addition of fludrocortisone.

It is important that patients on checkpoint inhibitors are informed about the potential danger of developing cortisol deficiency either due to pituitary or adrenal failure. Due to its relative novelty and rarity, checkpoint inhibitor-induced cortisol deficiency may go undiagnosed in patients presenting acutely to an emergency department; therefore, patients should be provided with documentation to present to healthcare professionals should they become unwell.

In common with other endocrine irAEs associated with checkpoint inhibitor therapy, the patient was able to continue on nivolumab therapy without interruption, and similar to the other agents in this class, the assumption is that the hormone deficiencies are irreversible and that endocrine replacement therapy will be open-ended. He has not developed any further endocrinopathies. 
In conclusion, much remains to be understood about the mechanism and risk factors predisposing to endocrinopathy with checkpoint inhibitor therapy. The use of checkpoint inhibitors in the treatment of a variety of malignancies is being investigated, and as their use expands, it is inevitable that the number of patients experiencing endocrine irAEs increases. Death or significant morbidity due to acute adrenal crisis should be avoidable by increased patient education and awareness among healthcare professionals. This case illustrates that cortisol deficiency secondary to checkpoint inhibitor use can be the result of primary adrenal failure as well as the more common complication of ACTH deficiency secondary to hypophysitis.

\section{Declaration of interest}

P Lorigan has been reimbursed for advisory work by BMS, Merck, Amgen, Novartis, GSK, Roche. He has received Speaker fees from BMS, Novartis, Merck and also support for travel from BMS, Merck. None of the other authors have any conflicts of interest to declare.

\section{Funding}

This research did not receive any specific grant from any funding agency in the public, commercial or not-for-profit sector

\section{Patient consent}

Written informed consent was obtained from the patient for publication of the submitted article.

\section{Author contribution statement}

$\mathrm{H}$ Trainer wrote the initial draft of the article, co-ordinated with the other authors and prepared the final manuscript. The patient was jointly managed by Prof. Lorigan and Prof. Trainer, who along with Dr Higham participated in the preparation of the manuscript on the report and provided final comments on the manuscript. Dr Hulse provided expert opinion on all radiological investigations undertaken.

\section{References}

1 Hodi FS, O'Day SJ, McDermott DF, Weber RW, Sosman JA, Haanen JB, Gonzalez R, Robert C, Schadendorf D, Hassel JC, et al. 2010 Improved survival with ipilimumab in patients with metastatic melanoma. New England Journal of Medicine 363 711-723. (doi:10.1056/ NEJMoa1003466)

2 Topalian SL, Hodi FS, Brahmer JR, Gettinger SN, Smith DC, McDermott DF, Powderly JD, Carvajal RD, Sosman JA, Atkins MB, et al. 2012 Safety, activity, and immune correlates of anti-PD-1 antibody in cancer. New England Journal of Medicine 366 2443-2454. (doi:10.1056/NEJMoa1200690)

3 Melero I, Hervas-Stubbs S, Glennie M, Pardoll DM \& Chen L 2007 Immunostimulatory monoclonal antibodies for cancer therapy. Nature Reviews Cancer 7 95-106. (doi:10.1038/nrc2051)

4 Torino F, Corsello SM \& Salvatori R 2016 Endocrinological side-effects of immune checkpoint inhibitors. Current Opinion in Oncology $\mathbf{2 8}$ 278-287. (doi:10.1097/CCO.0000000000000293)

5 Postow MA, Chesney J, Pavlick AC, Robert C, Grossmann K, McDermott D, Linette GP, Meyer N, Giguere JK, Agarwala SS, et al. 2015 Nivolumab and ipilimumab versus ipilimumab in untreated melanoma. New England Journal of Medicine 372 2006-2017. (doi:10.1056/NEJMoa1414428)

6 Weber JS, Yang JC, Atkins MB \& Disis ML 2015 Toxicities of immunotherapy for the practitioner. Journal of Clinical Oncology 33 2092-2099. (doi:10.1200/JCO.2014.60.0379)

Received in final form 4 October 2016

Accepted 14 October 2016 